\title{
ర \\ EDITOR'S \\ CHOICE \\ Just health: on the conditions for acceptable and unacceptable priority settings with respect to patients' socioeconomic status
}

\author{
Kristine Bærøe, ${ }^{1}$ Berit Bringedal ${ }^{2,3}$
}

${ }^{1}$ The Ethics Programme, IFIKK, University of Oslo, Oslo, Norway ${ }^{2}$ Harvard Medical School, Boston, Massachusetts, USA ${ }^{3}$ The Research Institute, The Norwegian Medical Association, Oslo, Norway

\section{Correspondence to} Kristine Bærøe, The Ethics Programme, University of Oslo, IFIKK, Postboks 1020 Blindern, Oslo 0315 , Norway: kristine.baroe@ifikk.uio.no

Received 22 December 2010 Revised 17 February 2011 Accepted 2 March 2011 Published Online First 8 April 2011

\section{ABSTRACT}

It is well documented that the higher the socioeconomic status (SES) of patients, the better their health and life expectancy. SES also influences the use of health services - the higher the patients' SES, the more time and specialised health services provided. This leads to the following question: should clinicians give priority to individual patients with low SES in order to enhance health equity? Some argue that equity is best preserved by physicians who remain loyal to 'ordinary medical fairness' in non-ideal circumstances when health disparities persist; ie, doctors should allocate care according to needs only and treat everyone with equal regard by being neutral with respect to patients' SES. This paper furthers a discussion of this view by questioning how equitable needs relate to SES. To clarify, it distinguishes between four versions of 'healthcare need' and approaches an acceptable conceptualisation of the notion supported by Norman Daniels' theory on health equity. It concludes that doctors should remain neutral to patients' SES in cases in which several patients require the same health care. However, equitable health care requires considerations of the impact of socioeconomic factors (SEF) on patients' capacity to benefit from the care. Remaining neutral towards patients' SES in this respect does not promote equal regard. It follows that priority setting on the basis of SEF is required in fair clinical distribution of care, eg, through allocating more time to patients with low SES. In order to advance equity accurately, the concept of ordinary medical fairness should be amplified according to this clarification.

Although the exact causes of health disparities are difficult to identify, a strong correlation between various living and working conditions and health status is well documented. ${ }^{1}$ The pattern is equal in all countries, rich or poor: the higher the socioeconomic status (SES) of patients, the better the health and the higher the life expectancy; health prospects are distributed along a social gradient. On a societal level, health inequalities correlate to the social determinants of health, such as education, household income and employment. This is recognised worldwide, also in welfare states where equality is strongly held and access to health care is universal.

Not all health inequality is unjust; so why would we hold that inequalities in health distributed along a social gradient represent an injustice? An influential argument regarding this issue is put forward by Norman Daniels. ${ }^{2}$ Departing from on Rawls' account of social justice and our social obligation to protect people's opportunities, Daniels argues that a society has a social obligation to protect the health of its citizens because protecting health contributes to protecting individuals' fair share of the normal opportunity range. Daniels defines 'the normal opportunity range' as 'the set of reasonable plans of life a society's members could pursue' (given an otherwise just distribution of resources), and the plans are reasonable for an agent '...if the agent has the relevant talents and skills'. ${ }^{3}$

Consequently, if health is considered essential to the promotion of a normal opportunity range and is, therefore, essential to the idea of justice, inequalities in health caused by social, controllable structures that affect people's opportunities for normal functioning are then unjust, ie, there is health inequity. We protect the individual's fair share of the normal opportunity range through an appropriate health policy aimed at protecting normal functioning. This includes a just distribution of social determinants of health as well as a just distribution of health care.

While correlations between social determinants and health status are well documented, we do not know much about how and to what extent healthcare personnel affect healthcare inequalities through their distribution of health care. Empirical research shows, however, that SES also correlates with the use of health services: the higher the SES, the more specialised the health services. ${ }^{4-7}$ The higher the SES, the more time spent with the patient. ${ }^{8}$ Moreover, SES seems to have an effect on physicians' perception of the patient. ${ }^{9}$ In addition, SES influences doctor-patient communication. ${ }^{10}$ Although we cannot conclude how this affects the care that patients receive, these findings point towards the possibility that health inequalities are reinforced by the service doctors provide. This effect can be distinguished from other sources to inequalities-such as unequal access, availability of care, or unequal quality of care. Furthermore, it is documented that doctors disagree on the definition of low SES and the role of SES in clinical decisionmaking, ${ }^{11}$ and they have different attitudes towards their role as healthcare distributors when facing socioeconomically disadvantaged patients. ${ }^{12}$ Against this backdrop of empirical findings, the need to discuss and clarify doctors' potential roles in maintaining, reinforcing, or reducing health inequity emerges.

Samia Hurst ${ }^{13}$ addresses the matter by asking, 'How is fairness in medicine best served, by remaining neutral to patients' social status in 
setting clinical priorities or by attempting to equalise unjust health inequalities by giving priority to the socially disadvantaged at the point of care'. Hurst's focus for discussion is a situation in which two patients need access to the same indivisible good. The question is whether a patient of low SES should be given priority in order to compensate for the socioeconomic disadvantage. Her main argument is that " $[\mathrm{t}] \mathrm{o}$ justify giving priority to low status, we must give priority to equal health over equal regard'. She concludes that 'we protect both equal health and equal regard by treating all alike according to need...'. She thus takes 'need' or, more precisely, 'medical need' as the proper criteria for priority setting and excludes, at least circumstantially, the idea that SES should be a criterion in deciding such matters.

Hurst's conclusion coincides with the concept she calls 'ordinary medical fairness', interpreted as a claim on neutrality with regard to patients' SES. This entrenched view on fairness is found in the Geneva Declaration and is accepted by the World Medical Association as a requirement to prevent irrelevant characteristics such as '....age, disease or disability, creed, ethnic origin, gender, nationality, political affiliation, race, sexual orientation, social standing or any other factor' from intervening between doctors' duty and their patients. ${ }^{14}$

Norman Daniels ${ }^{3}$ agrees that doctors should not base clinical priority settings on social worth. He argues that they are likely to do less harm if they address healthcare needs as encountered without filtering through concerns for social justice. However, he also states that he would not oppose affirmative action on the basis of class in certain contexts of non-ideal circumstances, just as he accepts affirmative actions on the basis of race and gender in certain contexts. Daniels ${ }^{3}$ states, however, that it is a challenge to distinguish acceptable affirmative actions on the basis of class from non-acceptable clinical priority settings based on social worth judgements.

We pick up this challenge. By questioning the general claim on neutrality towards patients' SES we wish to broaden the discussion about healthcare professionals' obligations in promoting health equity. We agree with Hurst's conclusion that equitable care requires assigning equal importance to the health outcome of all, ${ }^{13}$ and that SES alone cannot justify prioritising patients of low SES among others with similar health conditions. However, if we define 'ordinary medical fairness' on the basis of need without probing into the conditions that constitute equitable interpretations of need, we run the risk of hiding how SES should come into consideration in clinical care. Our aim is therefore to disclose such conditions and to identify the relevant way that socioeconomic factors (SEF) play a justified role in fair priority setting. Spelling out this role to healthcare professionals may bolster against the reinforcement of health inequity within health care caused by ignorance. We base our discussion on Daniels' theory of health equity ${ }^{2}$ and the assumption that 'need' should be more clearly definedaccording to the initial health condition and/or to the health outcome. We close the argument by considering the clinical and political consequences of our analysis.

\section{CLARIFYING ASSUMPTIONS}

In the following section, we explore the interpretation of 'need' involved in the proposed conceptualisation of 'ordinary medical fairness' as an equitable distribution of clinical care on the basis of need. According to Daniels' theory of health equity, ${ }^{2}$ how should 'healthcare need' be conceptualised in this situation?

While assuming non-ideal circumstances in which social health inequalities persist, the analysis relies upon a principle derived from Daniels' theory of the social obligation to protect equality in the normal range of opportunities: the principle of the social obligation to level up the health of those worst-off and, more precisely, level it up to the better-offs' level of normal functioning over a lifespan $(\mathrm{P})$.

In the following section, we consider SEF, such as education, income, profession, unemployment and housing, as part of what constitute SES.

\section{TOWARDS AN EQUITABLE NEED CONCEPT}

As is well documented, the concept of 'need' is ambiguous. ${ }^{15}$ Therefore, we do not base our discussion on a definite definition of 'healthcare need', although we accept the description as a 'condition-treatment pairing'. ${ }^{16}$ We approach a normative interpretation of the notion by considering two distinct dimensions of 'healthcare need' against the background of the normative premise above, P. These dimensions concern:

1. Whether it is the patient's actual health condition or a certain health outcome that should be the primary source to determine the equity of a specific distribution. In the first case, need corresponds to the means that are known usually to improve the actual condition independent of considerations of individual capacity to benefit from the treatment. Two patients, $A$ and $B$, can be said to have equal needs when their health conditions are the same. In the second case, the need correlates with the means taken to improve health according to a certain outcome. In this case, consideration of individual capacity to benefit from treatment is an essential part of the determination of the need. This means that if patients $A$ and $B$ have similar health conditions, but patient $B$ lacks the capacity to benefit from standard treatment, this patient has a different need than patient A.

2. Factors considered relevant when assessing needs. A definition of healthcare needs should clarify the relevant generic characteristics of patients and their situations that are involved. What we are interested in exploring in this paper is whether SEF should be included in the set of conditions that may constitute a healthcare need.

The answers to (1) and (2) have an impact on the distributive decision. Is there a legitimate reason to offer unequal amounts of resources/treatment to two patients with equal health states but different SES? If need is the basic criterion for a fair distribution, the ambiguity in the concept with respect to these two dimensions should be analysed and explicitly clarified.

The two different focuses on health condition or health outcome can be combined with the two possibilities of inclusion or exclusion of SEF in the definition of healthcare needs. This gives us four alternatives for conceptualising 'healthcare need' with regard to SES (see table 1). In the following we discuss how equity is pursued by these alternatives against the background of Daniels' theory on health equity. ${ }^{2}$ Daniels' theory on equal opportunities thus provides the justification of how 'healthcare need' should be conceptualised with regard to SES in a fair distribution of health care.

\section{Healthcare need, exclusion of SEF and equity}

On the assumptions of $\mathrm{P}$, the principle of the social obligation to level up the health of those worst-off, we will argue that (a) and

Table 1 Dimensions of health care needs

\begin{tabular}{lll}
\hline $\begin{array}{l}\text { Combinations of dimensions } \\
\text { constituting 'healthcare needs' }\end{array}$ & $\begin{array}{l}\text { Exclusion } \\
\text { of SEF }\end{array}$ & $\begin{array}{l}\text { Inclusion } \\
\text { of SEF }\end{array}$ \\
\hline Health condition focus & a & c \\
Health outcome focus & b & d \\
\hline
\end{tabular}

SEF, socioeconomic factors 
(b) are inequitable. The exclusion of SEF in the definition of need will not satisfy the social obligation to level up the worst-offs in terms of SEF. This is valid in both cases whether we consider need relative to health condition or health outcome.

Let us first consider how the exclusion of SEF in combination with focus on health condition (a) is incompatible with P. In this need concept, there is no room for considering whether the disadvantages of the socioeconomic worst-offs (for instance, lack of knowledge, bad economy, or deprived housing) may affect their capacity to benefit from care. Consequently, in cases in which SEF actually have an impact on individuals' capacity to benefit, the healthcare service will provide suboptimal care to these patients compared with those who get the same treatment without experiencing socioeconomic hindrances to benefit from treatment.

Moreover, if the doctor is unaware or ignorant of the potential impact that socioeconomic better-offs may have on the healthcare service in terms of their already acquired social advantages - say, high education - to communicate and claim their needs for health care, the socioeconomic worst-offs might end up getting comparatively less care and experience less health gain due to their lack of such relevant skills. Consequently, an ethical practice that merely focuses on health condition, and ignores SES and SEF, is likely to maintain or reinforce health inequity by the healthcare distribution itself. In cases in which SEF actually affect the outcome of treatment, patients of low SES will gain less health than better-offs with a similar health condition, ie, an assumed similar need. According to Daniels' theory on health equity ${ }^{2}$ and $\mathrm{P}$-the social obligation to level up the health of those worst-off to the level of the better-offs of normal functioning over a lifespan-a distribution of care based upon this understanding of 'healthcare need' is not equitable.

Let us now consider the case in which SEF are ignored in conjunction with the focus on health outcome (b). In cases in which SEF, and not health conditions, are the actual hinder to benefitting from treatment, a doctor who interprets healthcare needs this way has two options: (1) she or he can claim the case is futile (as SEF should be ignored) or (2) she or he can reconsider the patient's need (without paying attention to the possible impact of SEF) at every level of suboptimal outcome the patient achieves due to SEF until the expected health outcome is obtained.

Option (1) would be absurd - and also ethically unjustifiedif another effective option for care that circumvents the negative impact of SEF is available. Then, the case is indeed not futile. Option (2) is feasible, but ineffective in so far as alternative interventions that could circumvent the negative impact of SEF and bring the patients directly to the otherwise expected health level could be provided at the very start of the intervention. Let us illustrate this by an example. A woman with four children, two badly paid jobs, and an alcoholic husband is asked to start exercising in order to reduce her hypertension. This is the doctor's preferred intervention before giving her medication for her high blood pressure. In this case, the socioeconomic barriers represent a hindrance to complying with the advisory treatment. This failure may further expose her to stigma within the health system, rendering the advisory treatment even more ineffective. Ignorance of these barriers implies a longer time for recovery and poorer health over time compared with others who are not subjected to the same non-medical barriers. A more effective intervention would be to take into consideration how SEF influence the possibilities of benefitting from certain kinds of interventions.
Consequently, an avoidable step-by-step treatment procedure due to the impact of SEF reinforces health inequality. Again, reinforced health inequality would not be aligned with $\mathrm{P}$ and 'healthcare need' shaped by excluding the considerations of SEF in conjunction with either a health condition or a health outcome focus cannot be considered equitable.

\section{Healthcare need, inclusion of SEF and equity}

We proceed by exploring how healthcare distribution that takes SEF into account (c and d) may support an equitable distribution according to $\mathrm{P}$.

We first consider the interpretation of 'healthcare need' that allows SEF to be included in conjunction with a focus on health condition (c). We assume it is irrelevant whether the health condition is caused by SEF or not; if people are sick, what counts is their health condition. The problem we face now is that a focus on the initial health condition does not offer any way to determine how SEF have a relevant influence on people's needs. A 'healthcare need' interpretation that is insensitive to how SEF relate to health, but at the same time confer such factors some relevance, actually allow for both pro-high-SES or pro-low-SES discrimination among patients. Consequently, this interpretation of 'healthcare need' is obviously not aligned with $\mathrm{P}$ and must then be rejected as an appropriate interpretation to basing equitable distribution of health care.

As we now have rejected the three previous alternatives of 'healthcare need' to be counted as equitable, we are left with the fourth alternative, (d). Will the inclusion of SEF in the definition of 'need' when we focus on health outcome accord better with how equity is protected by $\mathrm{P}$ ?

This fourth alternative defines the relevance of SEF in healthcare need considerations according to outcome. Compared with the outcome of the treatment of similar medical cases in which SEF do not constitute barriers, SEF emerge as relevant concerns in a healthcare need concept if they have a negative impact on the otherwise expected outcome of an intervention. That is, if SEF reduce the benefit from a treatment in order to reach the otherwise expected health outcome, health care that circumvents or counterworks this effect of SEF should be considered to meet the needs.

In an empirical setting, this interpretation of how SEF affects need may avoid the reinforcement of health inequity by the healthcare service. When efforts are made to ensure that the potential health outcome is obtained effectively in terms of interventions that avoid socioeconomic obstacles and unnecessary delays (as in a step-by-step procedure), the health status of the patient will be levelled up for a longer period of time than the standard treatment would otherwise allow for. The distribution of health care on the basis of this fourth interpretation of 'healthcare need' may contribute to improving the health status of the socioeconomic worst-off relative to the better-off and, thus, reduce health inequity. Therefore, the inclusion of SEF in this meaning is on a par with $\mathrm{P}$.

\section{Towards a SEF and outcome-sensitive conceptualisation of 'healthcare need'}

A conceptualisation of healthcare needs to base a fair distribution on would have to specify the 'conditions' that can be involved. Our analysis suggests one such condition (among others we have not considered here) that should be included, namely the category 'SEF which affect the capacity to benefit from treatment and reach the outcome otherwise expected'. This element in a 'healthcare need' concept is consistent with the social obligation to level up the health of the socioeconomic 
worst-off to the better-offs' level of normal functioning over a lifespan. Consequently, in non-ideal circumstances in which social health inequalities persist, an equitable distribution of health care according to need (as suggested by Hurst and Daniels) ${ }^{313}$ must consider SEF in this respect.

\section{Implications for clinical and political priority settings}

It follows from the above that doctors would be obligated to search actively for socioeconomic barriers to effective treatment. In this sense, complete neutrality with regard to SEF and, thereby, SES cannot be required to promote a fair distribution of care. A patient's SES should trigger extra awareness and prompt efforts to discover whether there are relevant barriers in terms of SEF and how these should be circumvented in order to obtain effective treatment. Examples of such efforts could be alternative interventions, more detailed explications, information and advice about health-related issues, or welfare benefits. Allocating more time in order to determine the patient's needs with respect to SEF may also be considered. More research into barriers people experience with respect to SEF when it comes to benefitting from treatment is necessary to avoid inequity based on SES. Clearly, these suggestions indicate that priority setting with regard to SEF on a clinical and political level is required when care is distributed according to equitable needs.

\section{Revising the claim on neutrality in 'ordinary medical fairness'}

Hurst's defence of SES neutrality is based on the view that prioritising patients in clinical care on the basis of their SES, ${ }^{13}$ other things equal, is not justified. She discusses cases in which patients with different SES have identical needs for treatment, but limited resources exclude the possibility that all can get the indivisible good (such as the operating room). Using SES as a criterion for choosing between patients is hard to justify for several reasons: First, as pointed out by Hurst, ${ }^{13}$ we lack knowledge about the effects of such levelling-up health strategies. Therefore, prioritising patients with low SES resembles more retribution for social injustice in how basic institutions shape social determinants of health rather than purposive redistribution of care. Moreover, the effects of such a policy would occur as nothing but yet another version of discrimination, rationing care to the socioeconomic better-offs simply because of their status. To let arbitrary individuals in need carry the burden of social injustice cannot be justified, neither in terms of high nor low SES. In this respect, we agree with Hurst ${ }^{13}$ and Daniels $^{2}$ that neutrality towards SES is required. What our discussion suggests, however, is that the inclusion of SEF in need considerations, when this has an impact on beneficial gains, is on a par with an equitable clinical healthcare distribution. In this respect, neutrality towards SEF (and, implicitly, SES) is not justified. An adequate normative understanding of 'ordinary medical fairness' should distinguish between situations in which SEF represent necessary information to realise the intended outcome, and situations in which SEF-based priority represents discrimination for morally unacceptable reasons.

\section{CONCLUSION}

Based on Daniels' theory on health equity, ${ }^{2}$ we have shown that complete neutrality towards SES cannot consistently be claimed for an equitable distribution of clinical care according to need. Attention to SEF in individual patient care is required in so far as
SEF represent barriers to effectively gaining benefit from otherwise adequate treatment. This view contrasts with the entrenched view on 'ordinary medical fairness', which is presented so as to claim neutrality with regard to patients' SES. While SEF may serve as criteria for prioritising patients in order to ensure effective treatment for all, we have also argued that prioritising on SES alone is unacceptable. Therefore, we provide a theoretical answer to Daniels' challenge to find a way to distinguish acceptable from unacceptable priority setting based on patients' SES. However, we leave it to further research to make this distinction visible in real-life clinical care. Optimistically, this amplification of 'ordinary medical fairness' would involve that healthcare professionals increase their awareness of the relevance of SEF in clinical health care. This could help to avoid the reinforcement of health inequity in healthcare services by inaccurate interpretations of 'healthcare need' and biased care due to unconscious influence by patients' SES.

Acknowledgements The authors are grateful for valuable comments on earlier drafts of this manuscript from the reviewer of the journal, Samia Hurst, as well as from Norman Daniels, the research group Global Health: Ethics, Economics and Culture at the University of Bergen, and participants at the National Conference on Ethics at the University of Oslo, November 2010.

Funding KB is funded by the Ethics Programme, University of Oslo, Norway. BB is funded by the Commonwealth Fund, USA, and the Norwegian Research Council, Norway.

Competing interests None declared.

Provenance and peer review Not commissioned; externally peer reviewed.

\section{REFERENCES}

1. Wilkinson RG, Marmot M. Social determinants of health. the solid facts. Copenhagen: World Health Organization, 2003.

2. Daniels N. Just health: meeting health needs fairly. New York: Cambridge University Press, 2008:11-102.

3. Daniels N. Just health: replies and further thoughts. J Med Ethics 2009;35:36-41.

4. Hanratty B. How close have universal health systems come to achieving equity in use of curative services? A systematic review. Int J Health Serv 2007; 37:89-109.

5. van Doorslaer E, Masseria C. Income-related inequality in the use of medical care in 21 OECD countries. Paris: OECD Health Working Papers, No. 14, 2004. doi:10.1787/ 687501760705

6. Dunlop S, Coyte PC, Mclsaac W. Socio-economic status and the utilisation of physicians' services: results from the Canadian National Population Health Survey. Soc Sci Med 2000;51:123-33.

7. Grasdal A, Monstad K. Inequity in the use of physician services in Norway. Changing patterns over time? Bergen: Health Economics Working Papers, University of Bergen, 2010:5.

8. Gjelsvik R, Holmås TH, Monstad K. Socio-economic differences in treatment intensity in GP practice. HEB, særtrykk i helseøkonomi. Bergen: Rokkansenteret, 2009:17.

9. van Ryn M, Burke J. The effect of patient race and socio-economic status on physicians' perceptions of patients. Soc Sci Med 2000;50:813-28.

10. Willems S, De Maesschalck S, Deveugele M, et al. Socio-economic status of the patient and doctor-patient communication: does it make a difference? Patient Educ Couns 2005; 56:139-46.

11. Bernheim SM, Ross JS, Krumholz HM, et al. Influence of patients' socioeconomic status on clinical management decisions: a qualitative study. Ann Fam Med 2008;6:53-9.

12. Bringedal B, Bærøe K. Bør leger bidra til å utjevne sosial ulikhet i helse? [Should medical doctors contribute to reducing social inequality in health?] (in Norwegian). Tidsskr Nor Legeforen 2010;130:1024-7.

13. Hurst SA. Just care: should doctors give priority to patients of low socioeconomic status? J Med Ethics 2009;35:7-11.

14. World Medical Association. Declaration of Geneva. http://www.wma.net/en/ 30publications/10policies/g1/index.html (accessed 21 Dec 2010).

15. Culyer AJ, Wagstaff A. Equity and equality in health and health care. J Health Econ 1993;12:431-57.

16. Hasman A, Hope T, Østerdal LP. Health care need: three interpretations. J Appl Philos 2006;23:145-56. 